\title{
SELF-LOCKING AND OTHER MODES OF THE LASER OSCILLATIONS
}

\author{
L. LIS \\ Institute of Physics, Polish Academy of Sciences \\ Al. Lotników 32/46, 02-668 Warszawa, Poland \\ (Received August 10, 1993; in final form November 10, 1999)
}

In this paper experiments are presented on stability of laser generation at $632.8 \mathrm{~nm}$ for the $\mathrm{He}-\mathrm{Ne}$ tube with high amplifying coefficient. The experiments show that without any outer stimulation full stability of laser generation is obtained when self-locking is settled. Self-locking requires some conditions to be fulfilled for the cavity mirrors, i.e., their transmittivities and positions. The latter are the main subject of the experiments presented here. The results of an experiment show that in se lf-locking $\pi$ pulse is generated and forms swept absorption at $632.8 \mathrm{~nm}$ in the He-Ne discharge.

PACS numbers: 42.60 .FC

\section{Introduction}

Many experiments were performed on the mode locking of the $\mathrm{He}-\mathrm{Ne}$ laser at $632.8 \mathrm{~nm}$ in the sixties, see Refs. [1-7]. In general, the experiments concern the mode locking stimulated by passive or active intracavity loss modulation. However, there is also a possibility of the mode locking without any stimulation - the so-called spontaneous or self-locking (Refs. [3, 4] and [7]). The self-locking (SL) is obtained when cavity losses are above some critical value, however, well below the one above which the laser generation does not develop (Ref. [2]).

In Ref. [8] we also present some results of experiments on the $\mathrm{He}-\mathrm{Ne}$ laser at $632.8 \mathrm{~nm}$ in which the SL is achieved by partial screening of the laser beam inside the laser cavity. It is stated there that the SL is possible only when the distances of the cavity mirrors from the $\mathrm{He}-\mathrm{Ne}$ discharge are larger than critical ones. We assume that the latter are a function of the length of pulses generated by the laser system.

In this paper we continue with the question of cavity mirrors positions, and also with the laser system with resonant absorption. Instead of screening the intracavity radiation, the SL is obtained by the proper choice of transmissions of the mirrors, as shown in Ref. [2]. Also some experiments are presented on 
perturbation of the laser generation by some part of its radiation reflected back into the cavity. These experiments were mainly performed in order to estimate the real length of pulses generated in the SL and to compare it with the critical distances of the cavity mirrors.

\section{Experiments and results}

\subsection{Self-locking}

Generally, the experiments were performed in a system very similar to the one described in Ref. [8]. A laser tube with discharge capillary $150 \mathrm{~cm}$ in length and $3 \mathrm{~mm}$ of internal diameter was filled with isotopes ${ }^{3} \mathrm{He}$ and ${ }^{20} \mathrm{Ne}$ in the partial pressure ratio $10: 1$. The total pressure of the gases was fitted to obtain the maximum of the $632.8 \mathrm{~nm}$ laser generation. Methane cells with Brewster's windows stuck to the tube ends eliminated the laser generation at $3391 \mathrm{~nm}$ (see Ref. [9]). The discharge current was equal to $20 \mathrm{~mA}$ and was optimal for the laser generation.

A cavity was formed by high reflectivity mirrors of curvature radii 5 or $10 \mathrm{~m}$. The mirrors fixed on cardan gimbal mounts could be shifted to and from the He-Ne tube along a bench on which the tube was mounted.

The experimental set-up for investigations of the SL at various positions of the mirrors is shown in Fig. 1. $M_{+}$and $M_{-}$symbolize the mirrors forming the laser

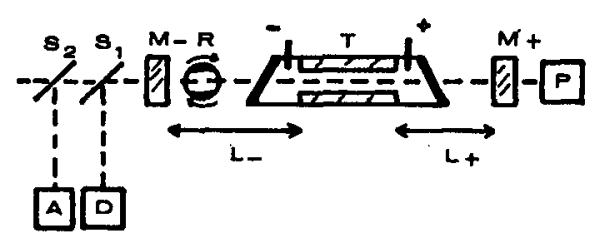

Fig. 1. Scheme of experimental system for investigations of laser generation for various positions of the cavity mirrors. $T$ : He-Ne tube, $D$ : laser output detection system, $A$ : spectrum analyzer system.

cavity; their distances from the positive and negative discharge ends are marked by $L_{+}$and $L_{-}$, respectively. Inside the cavity, between the mirror $M_{-}$and the tube, a mechanical chopper $R$ was used. The output radiation transmitted through the mirror $M_{-}$was directed by beam splitters $S_{1}$ and $S_{2}$ towards the fast photodiode and the spectrum analyzer, respectively. Current signals from the photodiode and the spectrum analyzer were registered with a Tektronix oscilloscope.

A small part of the radiation transmitted through the mirror $M_{+}$was registered with a power meter $P$. The power meter was mainly used for adjusting the cavity mirrors to the maximum of the laser generation after each mechanical operation on the laser system, in particular, after each shift of the mirrors. Spontaneous mode locking was obtained when total transmission of the cavity mirrors was not smaller than 7-8\%. In the experiments we used two mirrors: one with $6.6 \%$ and the other with $1.3 \%$ of transmittivity. The SL output through the mirror of $6.6 \%$ and $1.3 \%$ was 12 and $2.4 \mathrm{~mW}$, respectively. 
In Fig. 2 we have oscilloscope displays of the laser output interrupted periodically by the chopper $R$ for various distances of the mirrors $M_{+}(1.3 \%)$ and $M_{-}(6.6 \%), L_{+}$and $L_{-}$, respectively. Parts of the displays in the form of the belts
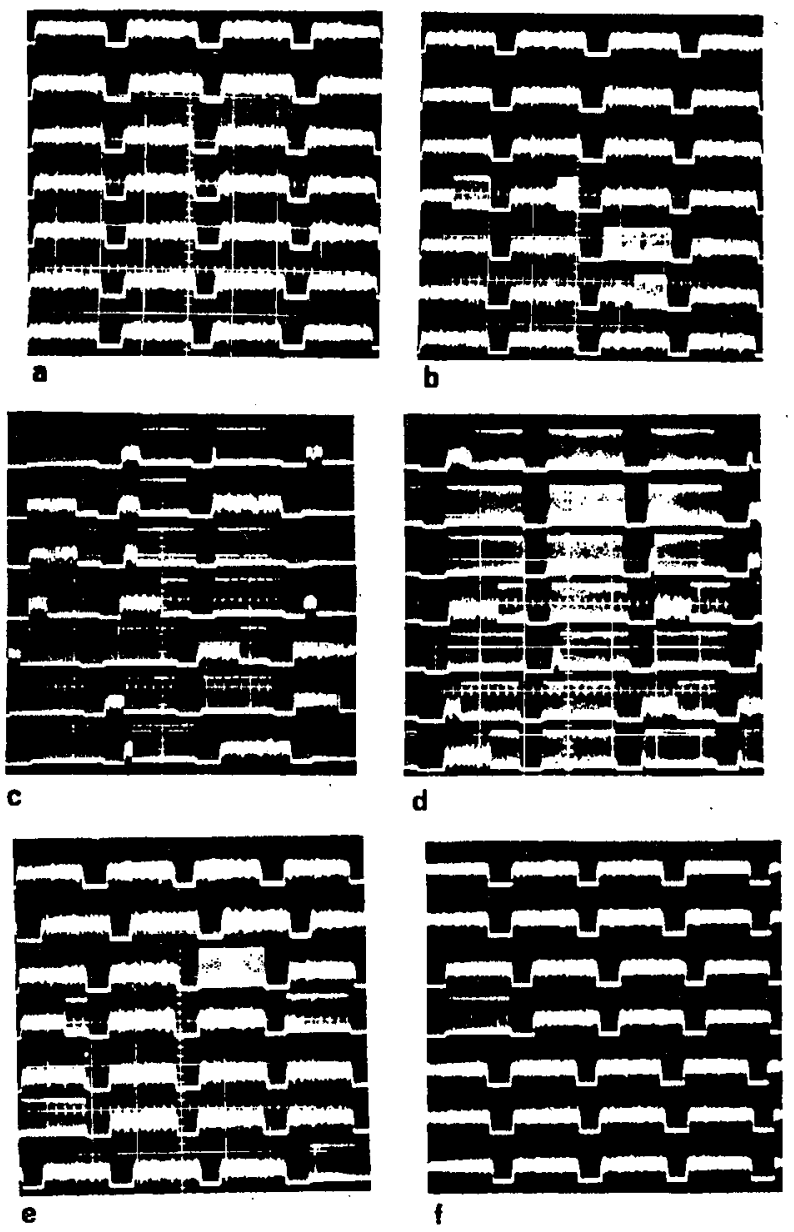

Fig. 2. Displays of the laser output for various mirrors distances $L_{+}$and $L_{-}$: (a), (b), (c), and (d) for $L_{+}=64 \mathrm{~cm}$ and $L_{-}$equal to $50,57,80$, and $95 \mathrm{~cm}$, respectively; and (e), (f) for $L_{-}=95 \mathrm{~cm}$ and $L_{+}$equal to 42 and $30 \mathrm{~cm}$, respectively. Oscilloscope sweep (OS): $10 \mathrm{~ms} / \mathrm{DIV}$.

signify the SL generation, whereas those in the form of chaotic spikes, the free running (FR) generation. Looking at the displays we may state that the FR appears simultaneously with resonator opening, whereas the SL only after some time. In other words, there is a stochastic time delay between the moment of the FR switching on and the SL appearance. (Here one may define a stability parameter or simply stability of the SL generation as the time after which the SL appears.) In Fig. 2 two series of displays of the laser output are shown: one for $L_{+}=64 \mathrm{~cm}$ and 
$L_{-}$changed from 50 to $95 \mathrm{~cm}$, Fig. 2a-d; and the other for $L_{-}=95 \mathrm{~cm}$ and $L_{+}$ changed from 64 to $30 \mathrm{~cm}$, Fig. $2 \mathrm{~d}-\mathrm{f}$. The best conditions for the SL are registered at $L_{+} \approx 64 \mathrm{~cm}$ and $L_{-} \approx 95 \mathrm{~cm}$. Further increasing of the mirror distances, over the values mentioned above, reduces stability. Also the SL is broken when $L_{+}$or $L_{-}$is decreased to 30 and $50 \mathrm{~cm}$, respectively. Below these distances (the critical ones) only the FR is observed.

As previously noted, the observations were made for the mirrors system of $M_{+}(1.3 \%)$ and $M_{-}(6.6 \%)$. Similar results are also obtained when the two mirrors replace each other, forming in this way a new cavity with $M_{+}(6.6 \%)$ and $M_{-}(1.3 \%)$. In this case, the critical distances of the mirrors show no difference and either of them equals about $50 \mathrm{~cm}$.

It is worth mentioning that in some range of the mirrors distances, close to the critical ones, laser generation jumps in an irregular way from the SL to the FR and vice versa.

\subsection{Additional feedback}

In Ref. [8] we suggested that the length of the pulses generated in the SL equals $40 \mathrm{~cm}$. Likewise in this experiment we should assume that the pulses outgoing through the mirror $M_{+}(1.3 \%)$ and $M_{-}(6.6 \%)$ have the lengths of 60 and $100 \mathrm{~cm}$, respectively $\left(2 \times L_{ \pm}\right.$critical one). Therefore, we wanted to find additional confirmation of the above suggestion in an experiment with an outer feedback with self perturbation as in Fig. 3 (see Ref. [6]). In the experiment some part of

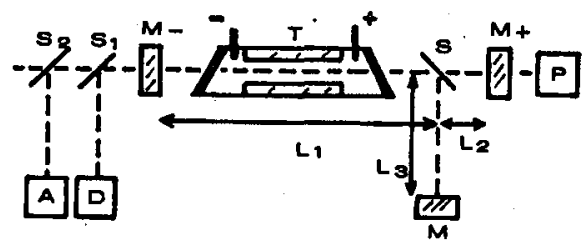

Fig. 3. Scheme of experimental system for investigations of laser generation perturbed by additional feedback.

laser radiation was extracted out of the laser cavity with a plate $S$ and reflected back with a mirror $M$. The mirror $M$ could be shifted along another bench, so that various distances of the mirror from the plate $S$ were available.

In Figs. 4-7 the results of the experiment are shown in dependence on $\Delta L=2\left(L_{3}-L_{2}\right)$, where $L_{3}$ and $L_{2}$ are distances of the plate $S$ from the mirror $M$ and $M_{+}$, respectively. The main result is the following: no perturbation of laser generation is observed when $|\Delta L|<3 \mathrm{~cm}$, see Fig. $4 \mathrm{a}$ and $4 \mathrm{~b}$. Changes of the signal seen at its top (see Fig. 4b) were artificially caused by light striking the gimbal mount of the mirror $M$. In another case (Fig. 4a) the mirror $M$ was mounted on a piezoelectric element driven by an alternating voltage generator $(A V)$. Oscillations of the mirror $M$ induced by the $A V$ generator helped in correct measuring of $\Delta L$ for which no perturbation was observed. Finally, it turned out that for $|\Delta L|=\left|\Delta L_{0}\right|=3 \mathrm{~cm}$ or a little more, the laser generation jumps from the SL 


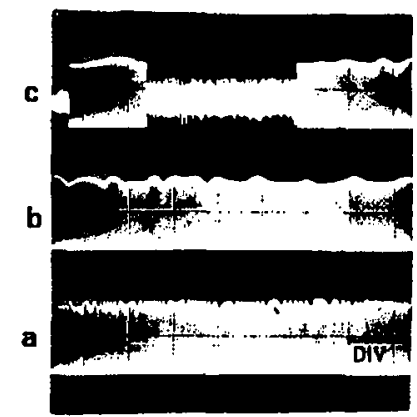

Fig. 4

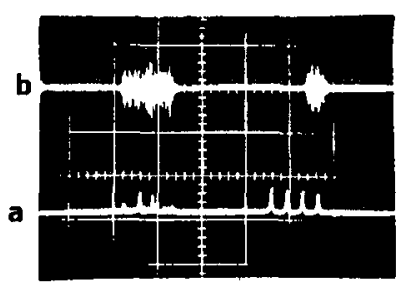

Fig. 5

Fig. 4. Displays of laser output for $\Delta L=0$, below but close to $3 \mathrm{~cm}$, and a little above $3 \mathrm{~cm}$ : (a), (b), and (c), respectively. OS: $10 \mathrm{~ms} /$ DIV.

Fig. 5. Displays of the spectrum and output of the laser: (a) and (b), respectively for $\Delta L=(1 / 2) L$. OS: $10 \mathrm{~ms} / \mathrm{DIV}$.

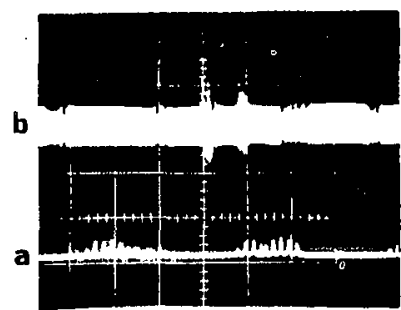

Fig. 6

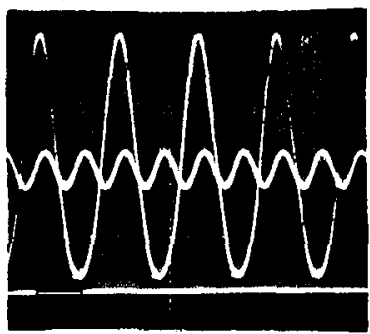

Fig. 7

Fig. 6. Displays of the spectrum and output of the laser: (a) and (b), respectively for $\Delta L=L$. OS: $2 \mathrm{~ms} / \mathrm{DIV}$.

Fig. 7. Display of the laser output from Fig. $6 \mathrm{~b}$ at fast oscilloscope sweep: $10 \mathrm{~ns} / \mathrm{DIV}$. The zero of both signals is displayed below as a straight line.

to the FR and vice versa, as is shown in Fig. 4c. Further increase in $|\Delta L|$, far beyond the range above, caused the FR or other states of the laser generation described in Ref. [6]. For instance, when $\Delta L=(1 / 2) L$, generation is obtained for which separation of the modes is four times larger than the separation for the basic resonator $L ; L=L_{1}+L_{2}$ (see Fig. 5a). A display of the laser output in the case above is shown in Fig. $5 \mathrm{~b}$ in which zero output signal is at the zero level of the spectrum shown in Fig. 5a. Another state of laser generation is obtained when $\Delta L=L$ (see Figs. 6 and 7 ). In this case, displays of the laser output and its spectrum are shown in Fig. $6 \mathrm{~b}$ and $6 \mathrm{a}$, respectively. And also as previously, zero of the laser output is at the zero level of the spectrum. Next, in Fig. 7 a display of the laser output from Fig. $6 \mathrm{~b}$ is shown at fast oscilloscope sweep, signal with small modulation amplitude, whereas the display with large modulation amplitude presents the SL without perturbation, when the mirror $M$ is screened. As you see, 
frequency modulation of the perturbed generation is twice as big as that of the unperturbed one.

\subsection{Neon discharge inside cavity}

Experimenting with the system, we also investigated how the resonant absorption, Ne discharge, inserted into the laser cavity affects the stability of SL generation. These investigations were performed with an experimental system as in Fig. 8. In the system a discharge tube filled with isotope ${ }^{20} \mathrm{Ne}$ (AT) was applied

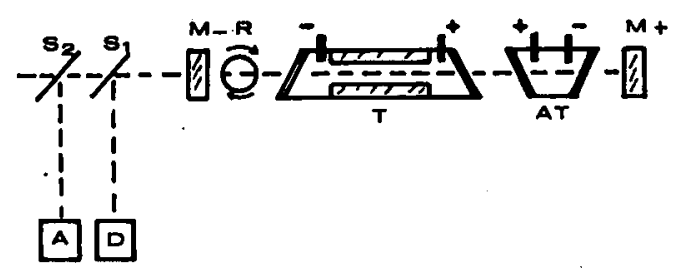

Fig. 8. Scheme of experimental system with the Ne tube inside the laser cavity. Symbols as in Fig. 1 and see the text.

as a resonance absorption element. The tube of $46 \mathrm{~cm}$ (and internal diameter of $8 \mathrm{~mm}$ ) discharge length was closed with Brewster's windows. Because of the $\mathrm{Ne}$ tube and its position inside the laser cavity, the minimal distance of the mirror $M_{+}$from the He-Ne discharge could be only $80 \mathrm{~cm}$. Here we present some results of the experiments for $L_{+}=82 \mathrm{~cm}$ and $L_{-}=105 \mathrm{~cm}$. The laser system at zero current in the Ne tube was active in the SL mode, though its stability was not so good as that in the best conditions. See display in Fig. 9a and compare it with that in Fig. 2c or 2d. Switching on the current of 20 or $30 \mathrm{~mA}$ in the Ne tube radically improves stability of the laser generation (Fig. 9b and 9c). However, further increase in the Ne tube current to 40 and next to $50 \mathrm{~mA}$ causes the lasering to jump from self-locking to one mode generation (Fig. 9d and in particular 9e).

In Fig. 10 the results obtained in the laser system described above, without the chopper $R$, are shown in two parts: the full self-locking signals, and their tops magnified five times. As is seen, the current in the Ne tube decreases the laser output, and also introduces some noise. The smooth signal at its top for zero current (Fig. 10a) gets fluctuations when the current in the Ne tube is increased to 30 and $45 \mathrm{~mA}$ (Fig. $10 \mathrm{~b}$ and $10 \mathrm{c}$, respectively).

Looking at Fig. 9b or $9 \mathrm{c}$ one may put a question if the Ne discharge causes the SL when $L_{-}$is below or equal to the critical distance? This question was the subject of investigations of the laser system with $L_{-}=50 \mathrm{~cm}$. As one can see, in such a system, without the Ne discharge, only the FR generation is developed (see Fig. 11a). However, switching on current in the Ne tube does not stimulate the SL but one mode generation only (Fig. 11b-e). The only case of the SL seen in one part of Fig. 11e seems to prove the rule mentioned above. 


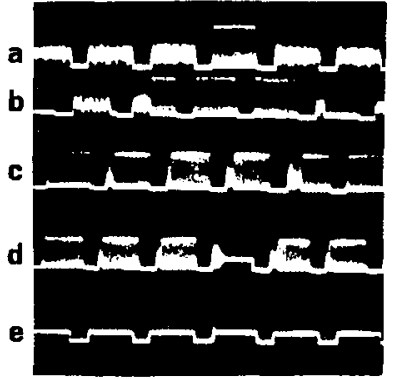

Fig. 9

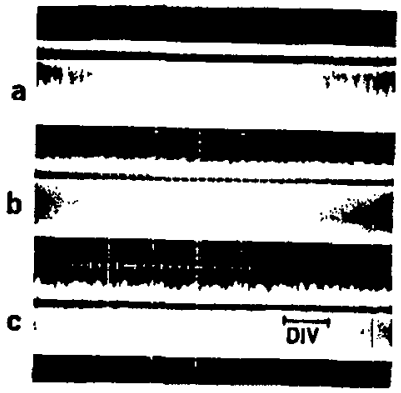

Fig. 10

Fig. 9. Displays of the laser output for the system with the Ne discharge tube and $L_{-}=105$ and $L_{+}=82 \mathrm{~cm}$. OS: $10 \mathrm{~ms} /$ DIV. Displays: '(a), (b), (c), (d), and (e) are for the Ne tube current $0,20,30,40$, and $50 \mathrm{~mA}$, respectively.

Fig. 10. Displays of the laser output for conditions as in Fig. 9 without chopper $R$. Displays: (a), (b), and (c) are for the Ne tube current 0,30 , and $45 \mathrm{~mA}$, respectively. The thin upper displays are five times magnified tops of the lower displays.

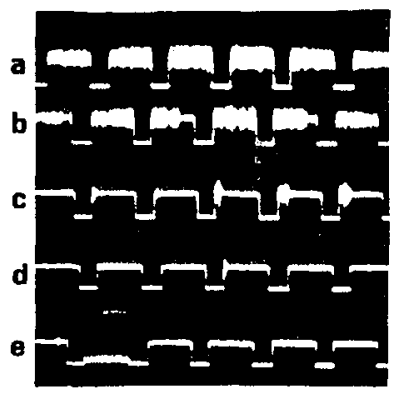

Fig.11

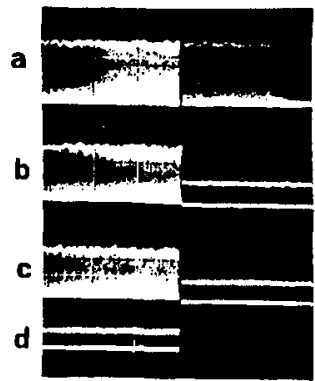

Fig.12

Fig. 11. Displays of the laser output for the system with the $\mathrm{Ne}$ tube and for $L_{-}=50 \mathrm{~cm}$ and $L_{+}=82 \mathrm{~cm}$. Displays (a), (b), (c), (d), and (e) are for the Ne tube current $0,20,35,40$, and $50 \mathrm{~mA}$, respectively. OS: $10 \mathrm{~ms} / D I V$. Displays: (a), (b), (c), (d), and (e) are for the Ne tube current $0,20,35,40$, and $50 \mathrm{~mA}$, respectively. OS: $10 \mathrm{~ms} / \mathrm{DIV}$.

Fig. 12. Hysteresis. Displays of the laser output for conditions as in Fig. 9. Displays (a), (b), (c), and (d) are for the Ne tube current 55, 60, 65, and $67 \mathrm{~mA}$, respectively.

\subsection{Hysteresis}

It is given that transitions between various states of the laser generation show hysteresis effects. Naturally, in the experiments of this work hysteresis effects were also observed many times, for instance, during transitions from the SL to one mode generation, and vice versa. Such an effect is shown in Fig. 12. The left side of the diagram shows laser output for the $\mathrm{Ne}$ discharge current increased from 55 to $67 \mathrm{~mA}$ (from top to bottom) (Fig. 12a-d). As we can see, the increase in the tube current to $65 \mathrm{~mA}$ decreases the SL output only. A little below, somewhere between 
65 and $67 \mathrm{~mA}$, the SL suddenly leaps into one mode generation (see Fig. 12d). Likewise, in the right side we see how intensity of this one mode generation is increased when the tube current is decreased back from 67 to $55 \mathrm{~mA}$. And now, one mode generation leaps into the SL, however, only when the tube current reaches about $55 \mathrm{~mA}$.

\section{Discussion}

Let us focus our attention on a large discrepancy between $\Delta L_{0}$ and the critical distances (the former is of the order $6 \mathrm{~cm}$ and the latter are 60 and $100 \mathrm{~cm}$ ). To understand this discrepancy let us analyze the experiment in detail with additional feedback. For this purpose, let us assume that the laser generates in the SL, which means a strong pulse running to and fro inside the cavity. The system $S M$ causes that, after or before the laser pulse, a small intensity pulse, an echo, is injected with the distance delay of $\Delta L=2\left(L_{3}-L_{2}\right)$ into the cavity. Theoretically, we have two possibilities: either the echo is damped completely in one loop in the cavity or not.

In the second case the echo, after one loop in the cavity, is gained by the next echo, and increasing in this way, after numerous round trips, begins to generate another echo, and so on. Hence we may conclude that inside the cavity an infinite train of echo pulses would appear if the SL had not been-destroyed.

In the first case, the echo disappears and the SL remains undisturbed. This occurs when $|\Delta L|<3 \mathrm{~cm}$. Complete damping of the echo inside the above mentioned range means its absorption by neon atoms that have been exposed to the action of the main laser pulse. Therefore, in view of the above, experiments on the additional \&ndback and cavity mirrors positions suggests that the laser pulse consists of two parts: a short spike, $\pi$ pulse or near $\pi$ pulse, shorter than $0.2 \mathrm{~ns}$ ( $0.5 \mathrm{~ns}$ is given in Ref. [2]), and a long tail (2-3 ns or more). Naturally, any increase in the intracavity laser radiation, by reducing the cavity losses, does not increase the spike but only increases the tail - its intensity and then its length. (Here one may define the length of the tail at the intensity of the laser radiation at which no perturbation of the opposite waves exists.)

Considering the SL generation one should also remember that there is a favorable cavity length for its stability connected with the time relaxation of the laser gain.

Now let us return to the case when the echo is increased and produces other echoes, that is, when $|\Delta L|>3 \mathrm{~cm}$. We find in Ref. [6] rough interpretation of the effects obtained in the case above. Then, as we know, the train of the echo pulses begins to develop - which generally leads to instability of the laser generation simply to the FR. However, when the $n$ echo, after running $m$ number loops inside the cavity, overlaps the laser pulse, the FR is not obtained; then a generation in a few frequency modes is settled. Stability of such generation is not perfect. See Figs. 5 and 6 . The condition above may be written as follows: $\Delta L: 2 L=m: n$ or $m \Delta \nu_{32}=n \Delta \nu$, where $n$ and $m$ are some integers and $\Delta \nu_{32}$ and $\Delta \nu$ are frequency separations of the modes for cavities with lengths $L_{3}-L_{2}$ and $L$, respectively.

The observed asymmetry of the critical distances $L_{+}$and $L_{-}$, in our opinion, is caused by two factors: the asymmetry of the cavity losses, and the asymmetry of the gain in the He-Ne discharge. The asymmetry of the cavity losses means that 
the tail of the laser pulse running towards the mirror of larger transmission is longer than the tail running towards the mirror of lower transmission. The asymmetry of the laser gain, we think, consists in the fact that the He-Ne discharge increases the laser radiation more intensively in the current flow direction than in the opposite one.

The next conclusion is self evident: resonant absorption inside laser cavity radically improves stability of the laser generation, however, it also introduces some chaotic changes in the intensity of the generated pulse. It seems that these changes are connected with too high neon pressure in the Ne tube.

However, the resonant absorption does not vary the critical distances; in our case $L_{-}$. For $L_{-}$below $50 \mathrm{~cm}$ no self-locking is observed even when the current of the Ne discharge is radically increased. In this case, at some current in the $\mathrm{Ne}$ tube free running generation leaps right into one mode generation.

\section{Conclusions}

Without any outer stimulation, and for the amplifiers with high gain, the self locking is the only state of the stable oscillation to which laser generation tends. Assuming that with maximum stability of the resonator and gain of amplifier, instability of laser generation appears when the opposite waves can be resonantly gained simultaneously (interference effects of the resonance interaction between atoms and radiation). For linear resonator avoiding of such opposite waves is possible only when laser generation develops in short pulses running inside the laser cavity. Naturally, the intensity of laser radiation beyond the pulse has to be negligible, which means that such radiation, forming the opposite waves inside the amplifier, should not involve any instability of laser generation.

The final conclusion is the following: the results of the experiment with additional perturbation prove that the short part of the pulse generated in the SL is $\pi$ or near $\pi$ pulse (see Refs. [7] and [10,11]), which produces swept absorption at $632.8 \mathrm{~nm}$ or, in other words, swept negative inversion of the population of the neon levels $5 s^{\prime}[1 / 2]_{1}^{0}$ and $3 p^{\prime}[3 / 2]_{2}$.

\section{References}

[1] R.E. McClure, Appl. Phys. Lett. 7, 148 (1965).

[2] M.H. Crowell, IEEE J. Quantum Electron. QE-1, 12 (1965).

[3] F.R. Nash, IEEE J. Quantum Electron. QE-3, 189 (1967).

[4] T. Uchida, A. Ueki, IEEE J. Quantum Electron. QE-3, 17 (1967).

[5] V.P. Chebotayev, I.M. Baterov, V.N. Lisitsyn, IEEE J. Quantum Electron. QE-4, 788 (1968).

[6] J. Hirano, T. Kimura, IEEE J. Quantum Electron. QE-5, 219 (1969).

[7] P.W. Smith, IEEE J. Quantum Electron. QE-3, 627 (1967).

[8] L. Lis, Nuovo Cimento D 11, 1377 (1989).

[9] L. Lis, Nuovo Cimento D 5, 451 (1985).

[10] A.G. Fox, P.W. Smith, Phys. Rev. Lett. 18, 826 (1967).

[11] J.D. Harvey, R. Leonhardt, P.D. Drummond, S. Carter, Phys. Rev. A 40, 4789 (1989). 